\title{
Acoustic Doppler velocimeter-induced acoustic streaming and its implications for measurement
}

\author{
C. M. Poindexter · P. J. Rusello $\cdot$ E. A. Variano
}

Received: 26 May 2010/Revised: 19 October 2010/Accepted: 23 October 2010/Published online: 16 November 2010

(C) The Author(s) 2010. This article is published with open access at Springerlink.com

\begin{abstract}
The acoustic Doppler velocimeter (ADV) is widely used for the characterization of fluid flow. Secondary flows ("acoustic streaming") generated by the ADV's acoustic pulses may affect the accuracy of measurements in experiments with small velocities. We assessed the impact of acoustic streaming on flow measurement using particle image velocimetry. The probes of two different ADVs were successively mounted in a tank of quiescent water. The probes' ultrasound emitters were aligned with a laser light sheet. Observed flow was primarily in the axial direction, accelerating from the ultrasound emitter and peaking within centimeters of the velocimeter sampling volume before dropping off. We measured the dependence of acoustic streaming velocity on ADV configuration, finding that different settings induce streaming ranging from negligible to more than $2.0 \mathrm{~cm} \mathrm{~s}^{-1}$. From these results, we describe cases where acoustic streaming affects velocity measurements and also cases where ADVs accurately measure their own acoustic streaming.
\end{abstract}

\section{Introduction}

The acoustic Doppler velocimeter (ADV) is a widely used tool for the characterization of fluid flow and turbulence. ADVs robustly measure three velocity components in a

C. M. Poindexter $(\bowtie) \cdot$ E. A. Variano

Department of Civil and Environmental Engineering,

University of California, Berkeley, CA 94720, USA

e-mail: cpoindexter@berkeley.edu

P. J. Rusello

DeFrees Hydraulics Laboratory,

School of Civil and Environmental Engineering,

Cornell University, Ithaca, NY 14853, USA small sampling volume at high temporal resolution (Lohrmann et al. 1994). Since their development in the 1990s, ADVs have been used in a diverse range of applications, such as turbulence measurements in the surf zone (Elgar et al. 2005) and estimation of vegetation-induced drag in wetlands (Nepf 1999).

Because an acoustic Doppler velocimeter measures velocity at a location at least $5 \mathrm{~cm}$ away from its probe tip, users and manufacturers regard the device as non-intrusive. However, deployment of ADVs in low-flow environments like wetlands may be hampered by a unique source of bias related to the ADV's mode of operation. The ADV operates by emitting ultrasonic pulses from a central transducer along a narrow beam. Two to four receiving transducers are spaced uniformly about the emitter and angled inward, defining a sample volume 5-18 $\mathrm{cm}$ away (depending on the ADV model). The receivers measure the return signal scattered by tracer particles in the sampling volume and compute the velocity from the shift in phase between a pair of acoustic pulses (Voulgaris and Trowbridge 1998; Lhermitte and Serafin 1984). Obtaining valid velocity measurements requires a high signal-to-noise ratio (SNR) in the acoustic backscatter. SNR depends on tracer particle density and ADV configurable settings such as transmitted acoustic power.

The transmitted acoustic beam can generate a steady flow in the direction of sound propagation in a process commonly known as acoustic streaming (and also referred to as steady streaming, quartz wind, Eckart streaming, or acoustic straight flow). Acoustic streaming is a largely unexamined source of ADV measurement bias that may particularly impact measurements in low flows. Evidence of this effect was reported by Snyder and Castro (1999), in which a Nortek acoustic Doppler velocimeter measured non-zero velocities up to $2 \mathrm{~cm} \mathrm{~s}^{-1}$ in still water. For flows 
perpendicular to the ADV probe's axis ("cross-flows") of $2 \mathrm{~cm} \mathrm{~s}^{-1}$ or higher, the phenomenon appeared to largely disappear.

Acoustic streaming, documented in the literature as early as 1831 (Faraday), stems from a gradient of sound energy density in the direction of sound propagation, a gradient set up primarily by the absorption of the emitted sound (Riley 1997). Several approximate analytical solutions for acoustic streaming induced by a narrow ultrasound beam exist (e.g. Makarov et al. 1989; Wu and Du 1993; Mitome et al. 1995; Riley 2001). A common approach uses the momentum equation for incompressible, viscous fluid with an external force field, $f$, to represent the driving force (Eq. 1).

$\frac{\partial \boldsymbol{u}}{\partial t}+\boldsymbol{u} \cdot \nabla \boldsymbol{u}=-\frac{\nabla p}{\rho}+\frac{\mu}{\rho} \nabla^{2} \boldsymbol{u}+f$

We adopt a coordinate system where the ultrasound beam axis defines the $z$-axis and the vertical (or axial) direction, and the $r$-direction extends radially outward from the beam axis. Within the narrow ultrasound beam penetrating the semi-infinite volume $(z>0)$, the time-averaged sound energy density, $\langle E\rangle$, at a distance $z$ from the emitter and integrated across the cross-sectional area of the beam is:

$\langle E\rangle=\frac{P}{c} \exp (-\beta z)$

$\beta$ represents the linear sound attenuation coefficient; $P$ is the emitter power, and $c$ is the speed of sound (Lighthill 1978). The linear attenuation coefficient follows from the simplifying assumption that sound amplitude does not affect the sound speed. The driving force $f$ is proportional to the gradient of the time-averaged sound energy density (Mitome et al. 1995):

$f=-\frac{1}{\rho} \frac{d\langle E\rangle}{d z}$

To derive analytical solutions for acoustic streaming velocity, $\boldsymbol{u}$, the nonlinear term in Eq. 1 is neglected, sometimes by appealing to the method of successive approximations (Nyborg 1998; Wu and Du 1993). These solutions indicate vertical streaming velocity on the ultrasound beam centerline $w_{r=0}$ is proportional to the square of the sound source amplitude, $a^{2}$, and hence directly proportional to the transmitted sound power, P (Nyborg 1998; Mitome et al. 1995; Wu and Du 1993). In practice, Reynolds numbers associated with any noteworthy acoustic streaming are too high to neglect the nonlinear term in Eq. 1 (Lighthill 1978; Kamakura et al. 1996). A scaling analysis assuming infinitely large Reynolds number indicated that streaming velocity is proportional to $a$ (and thus the square root of $P$ ) rather than $a^{2}$ (Mitome et al. 1995). Regardless, these results suggest streaming velocity depends strongly on transmitted power $P$. Transmitted power varies between ADV models, and between configurations of the same ADV model, and is an important mechanism by which ADV users can control the magnitude of acoustic streaming (see Sect. 4).

The available analytical solutions to Eqs. 1-3 also describe the variation of acoustic streaming velocity with distance $z$ from the ultrasound beam source. The streaming velocity along the ultrasound beam centerline, $w_{r=0}$, is negligible near the source and increases nonlinearly with distance (in the direction of ultrasound propagation) (Riley 2001; Mitome et al. 1995; Wu and Du 1993). Including the effect of radial momentum transport (transport away from the ultrasound beam axis) gives a solution in which $w_{r=0}$ increases nonlinearly, peaks and then begin to drop off substantially (Mitome et al. 1995).

The ultrasound transmitted by an ADV differs from the ultrasound considered in many theoretical analyses of acoustic streaming in that it is pulsed rather than continuous. Experimental data from tests of medical ultrasound devices suggest that whether sound is pulsed or continuous affects maximum streaming velocities and streaming velocity profiles (Starritt et al. 1989). Specifically, for the same time-averaged power emission, pulsed sound results in significantly increased streaming velocities overall and particularly near the emitter. This phenomenon relates to the frequency dependence of the sound attenuation coefficient, $\beta$, which in distilled water varies from $0.0023 \mathrm{~dB}$ at $1 \mathrm{MHz}$ to $23 \mathrm{~dB}$ at $100 \mathrm{MHz}$ (Kaye and Laby 1986). Hydrophone measurements of medical ultrasound equipment showed that pulsed sound leads to significantly more rapid harmonic formation than continuous sound (Starritt et al. 1989). Because sound absorption increases with sound frequency squared (Kuttruff 1991), more rapid harmonic formation leads to more rapid sound absorption, a steeper gradient in sound energy density, and increased streaming near the transmitter. To account for this effect, $\mathrm{Wu}$ and $\mathrm{Du}$ (1993) proposed an analytical solution for acoustic streaming velocity due to pulsed ultrasound. The solution takes the same form as the solution for continuous, non-pulsed ultrasound with two modifications. First, the streaming velocity is not a function of emitted acoustic power, which for pulsed sound varies in time. Instead, the velocity depends on the peak instantaneous acoustic power. Second, a duty factor equal to the ratio of pulse duration to pulse repetition period is included. This model predicts that when keeping time-averaged power transmission constant, lower duty factors lead to higher acoustic streaming velocities ( $\mathrm{Wu}$ and $\mathrm{Du}$ 1993). This is because low duty factors correspond to higher peak instantaneous power. For a typical ADV, duty factors range from 0.002 to 0.02 depending on the nominal velocity range setting (Atle Lohrmann, personal communication, 7/14/2009). 
Various techniques from hot-film anemometry to particle image velocimetry (PIV) have been used to characterize the acoustic streaming induced by medical ultrasound equipment (Starritt et al. 1989; Cosgrove et al. 2001; Choi et al. 2004), ultrasound sonochemical reactors (Kumar et al. 2007), and generic ultrasound transducers (Kamakura et al. 1996). To our knowledge, only the ADVs themselves have been used to measure ADV-induced acoustic streaming (Snyder and Castro 1999; Hartley 1995), giving a limited picture of the phenomenon. In order to fully characterize acoustic streaming induced by acoustic Doppler velocimeters, we investigated the flow field around two very different ADVs operating in quiescent fluid with PIV. We varied the ADV adjustable settings that determine duty factor and transmitted power to determine the extent to which ADV-induced streaming corresponds with existing acoustic streaming theory. With the aid of this theory and a background on the range of current ADV applications in the laboratory and the field, we examined the potential for acoustic streaming to interfere with accurate ADV velocity measurement.

\section{Methods}

We applied PIV to two different ADV models as they collected flow measurement data. Each ADV model is produced by a different manufacturer and designed for a different environment. The 4-receiver, $10-\mathrm{MHz}$ Nortek Vectrino (Nortek AS, Norway) has a sampling volume centered $5 \mathrm{~cm}$ from the ultrasound emitter and is intended for laboratory use. The 3-receiver, $10-\mathrm{MHz}$ SonTek ADVField (SonTek/YSI, San Diego, CA) samples over a volume centered $10 \mathrm{~cm}$ from the ultrasound emitter and is intended for field use. The probe of each ADV was mounted in a glass tank of quiescent water such that the ultrasound emitter was aligned with a laser light sheet (thickness $\sim 2 \mathrm{~mm}$ ). The light sheet was generated by a pulsed, 532-nm dual Nd:YAG laser (Quantel USA) followed by a series of lenses (Fig. 1). The Vectrino's probe was attached to a linear positioning slide. A tripod head held the linear positioning stage in place and allowed for leveling via pitch, roll, and yaw adjustments. To align the ADV emitter axis (which defines the $z$-direction in our coordinate system) with the laser light sheet, we advanced the slide in the direction perpendicular to the laser light sheet in 0.05 inch increments, evaluating the PIV-measured velocities at each location. Laser and emitter alignment was assumed to occur at the position yielding the largest vertical velocities as measured by PIV. Due to its larger size, the ADVField required a stronger mounting system that was less adjustable. Levels were used to set the ADVField's orientation, and the laser light sheet was

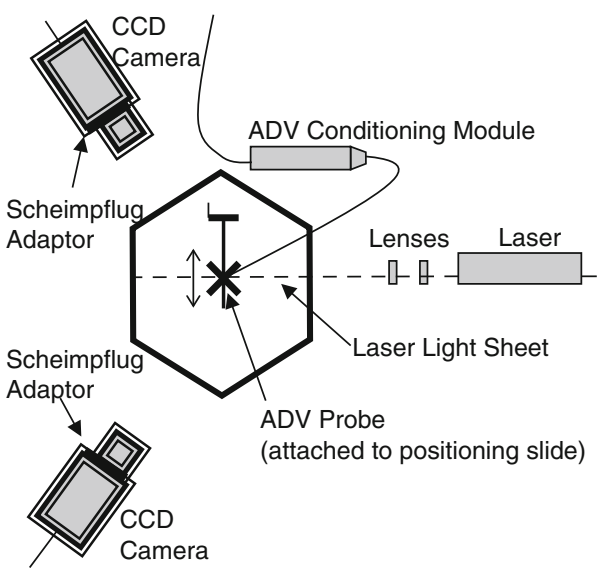

Fig. 1 Experimental setup, in which stereoscopic PIV resolves ADVinduced velocities; the $z$-axis is oriented normal to the page

positioned along the center of the emitter axis by eye. Built-in electronic levels were used to confirm that the ADVField's emitter was indeed level. Measurements of the radial dependence of streaming velocity, $w(r)$, for both ADVs suggested that this level of precision is adequate for resolving peak streaming velocities. Both the Vectrino and ADVField probes (except for the emit and receive transducers) were covered in black tape to minimize potentially damaging reflections.

Two-dimensional three-component (2D3C) PIV was performed. Compared to standard 2D2C PIV, this method can reduce errors due to misalignment between light sheet and calibration target. Such above-average PIV precision is useful in this study, given the small velocities that were measured. Each of two charge-coupled device (CCD), $1,600 \times 1,200$ pixel cameras (Imager Pro-X, LaVision, Gottingen, Germany), was oriented such that the lens paralleled one face of a hexagonal, 20-gallon glass tank (see Fig. 1). This configuration reduces errors from refraction by the glass. Scheimpflug adaptors on the cameras allowed for focusing over the entire field of view despite the oblique camera angle. Camera field of view was chosen to include the ADV transmitter and the sample volume and thus was typically less than $10 \mathrm{~cm}$ by $10 \mathrm{~cm}$ for the Vectrino measurements and greater than $10 \mathrm{~cm}$ by $10 \mathrm{~cm}$ for the ADVField measurements.

Municipal tap water added to the glass tank was first filtered using a 20-micron cellulose filter (Pentek) to minimize the number of large particles. Tracer particles (Sphericel, Potters Industries) with median diameter $11 \mu \mathrm{m}$ were added to the filtered tap water and the water then mixed with a submersible pump. After allowing currents generated by the pump to decay, we recorded multiple sets of images while the ADV was operating in the tank. Before recording each set of images, we modified one or more useradjustable ADV settings and initiated ADV data collection. 
Then, after a delay of more than $15 \mathrm{~s}$ (the maximum acoustic streaming start up time reported in Kamakura et al. 1996), we recorded 341 images in single-frame mode at $29.41 \mathrm{~Hz}$ using DaVis software (equivalent to a measurement duration of approximately $11.5 \mathrm{~s}$ ). The maximum number of images that could be recorded in a single set was determined by the RAM capabilities of the hardware. Sets of images were also recorded with the ADV off (no ADV data collection) before most measurements of ADVinduced streaming in order to establish the level of background flow in the tank.

We measured the flow induced by each ADV while systematically varying operating configurations. The Vectrino allows the duration, power, and repetition frequency of its ultrasound pulses to be configured (Table 1). No other userconfigurable setting was observed to affect acoustic streaming. The duration of each ultrasound pulse is specified by the "transmit length" while the pulse repetition frequency is specified using the "nominal velocity range" setting. A larger nominal velocity range setting corresponds to a higher pulse repetition frequency and higher duty factor. The Vectrino configuration is reset to a default configuration (Table 1) each time the Vectrino software restarts. Only the ADVField's pulse repetition frequency is typically adjusted, though it is possible to modify the pulse duration. There is no option to increase transmitted pulse power directly for the ADVfield. While testing the Vectrino, we held two of the three configurable settings that affect streaming velocity constant (at the level which caused the most acoustic streaming), while varying the remaining setting. We also varied multiple Vectrino settings at once. In total, we tested 16 different combinations of 120 different possible combinations of settings. The combinations of Vectrino settings we investigated are those yielding the greatest variation in acoustic streaming velocity. As is typical in field operation, only the nominal velocity range setting on the ADVField was adjusted during imaging. Repeat tests of the Vectrino in the same configuration spaced months apart confirmed that neither ADV set up, temperature, seeding density nor other unknown factors substantially influenced streaming velocities.

Velocity vector fields were computed from a time series of recorded images via stereoscopic cross-correlation in $\mathrm{DaVis}$ FlowMaster software. A multipass (or iterative) technique was used with an initial interrogation window size of $128 \times 128$ pixels or $64 \times 64$ pixels and a final interrogation window size of $32 \times 32$ pixels with $50 \%$ overlap between windows and Gaussian subwindow weighting. For the ADV configurations resulting in the slowest streaming velocities, computing vector fields from successive images (separated by $34 \mathrm{~ms}$ ) resulted in very small displacement (in pixels). In these cases, non-sequential images separated by up to $0.20 \mathrm{~s}$ were used. We imported vector field results to MATLAB for the analysis of maximum streaming velocities, mean velocities in the sampling volume, and along-beam velocity profiles. Measures of central tendency, computed over approximately $11.5 \mathrm{~s}$ (341 velocity fields), confirmed statistical convergence over the measurement period.

The ADV sampling volume is an irregular shape defined by the intersection of the transmitted ultrasound beam and the receive beams. The sampling volume may be approximated as a 6-mm-diameter circular cylinder for both the ADVField and the Vectrino (SonTek/YSI 2001; Nortek 2009). In contrast, our stereoscopic PIV measured velocities in a plane cutting through the center of sample volume. Each velocity vector represented a spatial average over a rectangular interrogation window within this plane. To compare the velocity vector fields obtained through PIV with the velocities measured by the ADV itself, we computed a weighted average of the PIV-measured velocity vectors falling within the ADV sampling volume. Each velocity vector was weighted according to the volume of the solid of revolution created by rotating the corresponding interrogation window about the sampling volume axis. The resulting estimates of sample volume average velocity $\left(w_{s}\right)$ approximate what the ADV itself measures, albeit with some uncertainty because the exact shape of the sampling volume is unknown. Velocity measurement uncertainties were estimated from PIV data as the larger of (a) the bootstrap 95\% confidence interval and (b) the interquartile range of vertical velocity over twelve PIV-based background flow measurements.

\section{Results}

Both the Vectrino and the ADVField-induced velocities were primarily in the $z$-direction, i.e., parallel to the ADV

Table 1 ADV user-selectable settings and their physical significance

\begin{tabular}{lllll}
\hline Setting name & Physical significance & Available settings-Vectrino & $\begin{array}{l}\text { Available settings- } \\
\text { ADVField }\end{array}$ & $\begin{array}{c}\text { Default settings- } \\
\text { Vectrino }\end{array}$ \\
\hline Nominal velocity range & Ultrasound pulse repetition & $\pm 0.03,0.10,0.30,1.00,2.50$, & $\begin{array}{c} \pm 0.03,0.10,0.30,1.00, \\
2.50 \mathrm{~m} \mathrm{~s}^{-1}\end{array}$ & $0.30 \mathrm{~m} \mathrm{~s}$ \\
& frequency & $4.00 \mathrm{~m} \mathrm{~s}^{-1}$ & NA & $1.8 \mathrm{~mm}$ \\
Transmit length & Pulse duration & $0.3,0.6,1.2,1.8,2.4 \mathrm{~mm}$ & High \\
Power level & Transmitted sound power & Low, Low,+ High-, High & NA & \\
\hline
\end{tabular}


Fig. 2 The acoustic output of a Nortek Vectrino induces a steady flow in tracer-particleladen, quiescent water as shown in a CCD camera image overlain by a PIV-generated vector field and colored according the vertical velocity magnitude

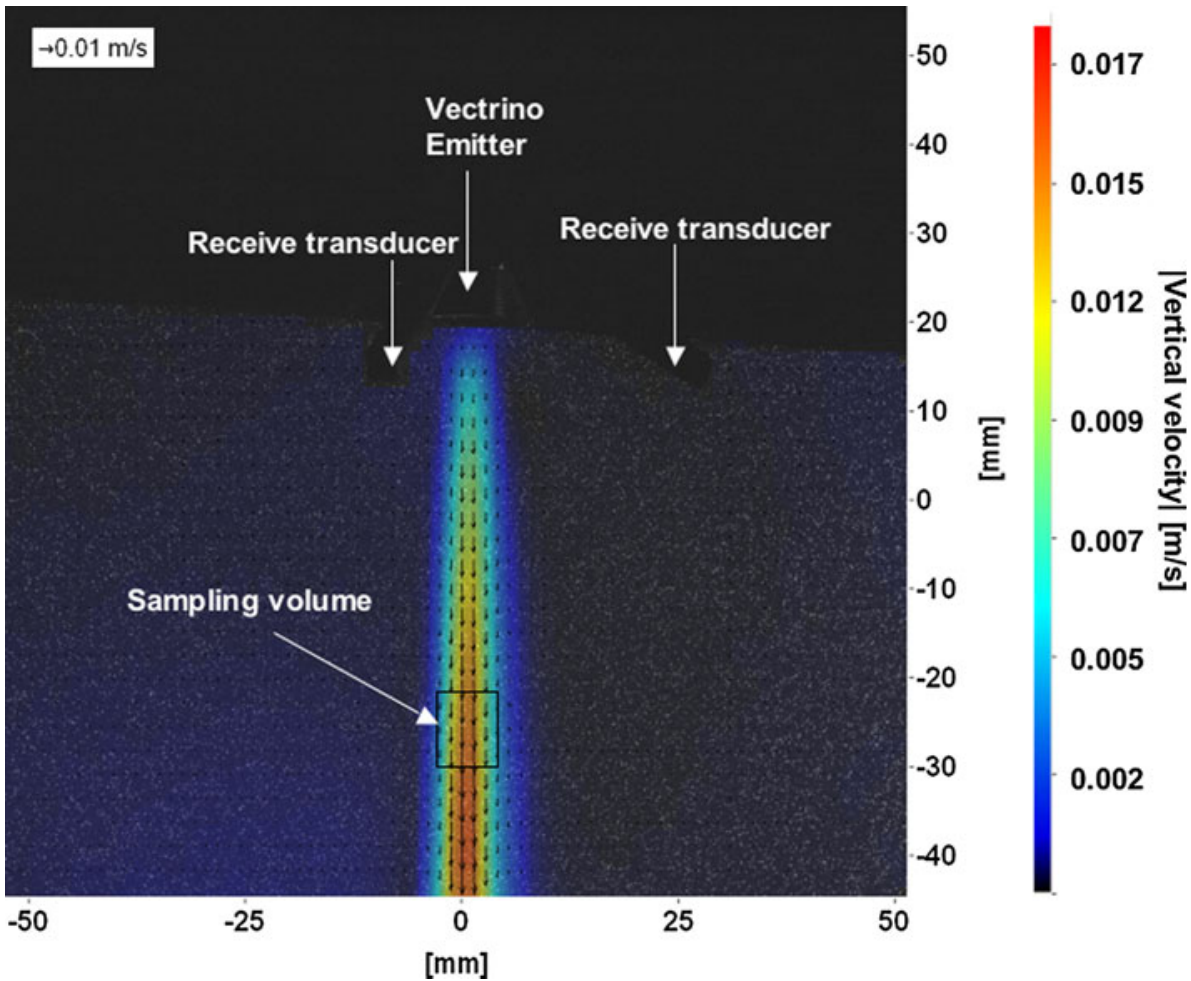

emitter axis (e.g. Fig. 2). The flow originated at the ultrasound emitter and increased with distance from the emitter, extending to the ADV sampling volume and beyond. Profiles of velocity along the centerline of the transmit beam, $w_{r=0}$ (Figs. 3, 4) show that for both ADVs, the velocity at the sample volume is close to the maximum velocity.

The Vectrino generated peak velocities $\left(w_{p}\right)$ between $0.05 \mathrm{~cm} \mathrm{~s}^{-1}$ and $2.0 \mathrm{~cm} \mathrm{~s}^{-1}$, while the ADVField generated peak velocities $\left(w_{p}\right)$ between $0.95 \mathrm{~cm} \mathrm{~s}^{-1}$ and $2.2 \mathrm{~cm} \mathrm{~s}^{-1}$. These peak velocities are aligned with the axes of the ADVs' ultrasound emitters (where $r=0$ ) and can be large enough to overwhelm the flow signal in some wetland, boundary layer, or backwater flows. Variation of the acoustic streaming velocity as a function of ADV configuration is seen in Table 2 in order of decreasing peak velocity. The greatest ADVField-induced velocities were observed with the nominal velocity range at $\pm 2.50 \mathrm{~m} \mathrm{~s}^{-1}$ (the largest available nominal velocity range setting). With the nominal velocity range set to the lowest available value, the ADVField generated velocities approximately $1 \mathrm{~cm} \mathrm{~s}^{-1}$ lower. The greatest Vectrino-induced velocities were observed with the following Vectrino settings: power level = high, transmit length $=2.4 \mathrm{~mm}$ (largest possible), and nominal velocity range $= \pm 4.0 \mathrm{~m} \mathrm{~s}^{-1}$ (largest possible). Smaller transmit lengths, nominal velocity ranges, and power levels resulted in slower acoustic stream velocities. The smallest Vectrino-induced flows $\left(w_{p}<0.5 \mathrm{~cm} \mathrm{~s}^{-1}\right)$ occurred when the power level was set to anything but "high" or when both the transmit length and nominal velocity range were reduced to their lowest level. Decreasing either the Vectrino's nominal velocity range or the transmit length in successive steps from the maximum to the minimum value produced a gradual monotonic decrease in the induced velocity (Fig. 3a, c). In contrast, adjusting the power level has a strong "stepfunction" type response; adjusting the power level from its highest to second highest setting ("high" to "high-") drastically decreased peak velocities (from $w_{p} \approx 2$ to $w_{p} \approx 0.25 \mathrm{~cm} \mathrm{~s}^{-1}$ ) (Fig. 3b). The ADVField behaved similarly to the Vectrino in response to reductions in nominal velocity range; induced velocities dropped by a fraction of a $\mathrm{cm} \mathrm{s}^{-1}$ each time the range was lowered (Fig. 4). In general, the distance to the maximum streaming velocity from the ultrasound emitter increased with increasing nominal velocity range, increasing transmit length and increasing power level.

To understand the effect of acoustic streaming on the measurements reported by the ADV itself, the PIV data were used to compute the average velocity over the sampling volume $w_{s}$. The values of $w_{s}$ are smaller than the peak velocities $w_{p}$, but follow the same trends relative to ADV configuration. The maximum $w_{s}$ value was $1.42 \mathrm{~cm} \mathrm{~s}^{-1}$ for the ADVField and $0.87 \mathrm{~cm} \mathrm{~s}^{-1}$ for the Vectrino (Table 2). Sampling volume average velocities increase with increasing nominal velocity range for both the Vectrino and ADVField (Fig. 5).

The differences between $w_{s}$ and $w_{p}$ stem in part from the sampling volumes' location with respect to peak-induced flows (visible in Figs. 3, 4). The sample volume is fixed in 

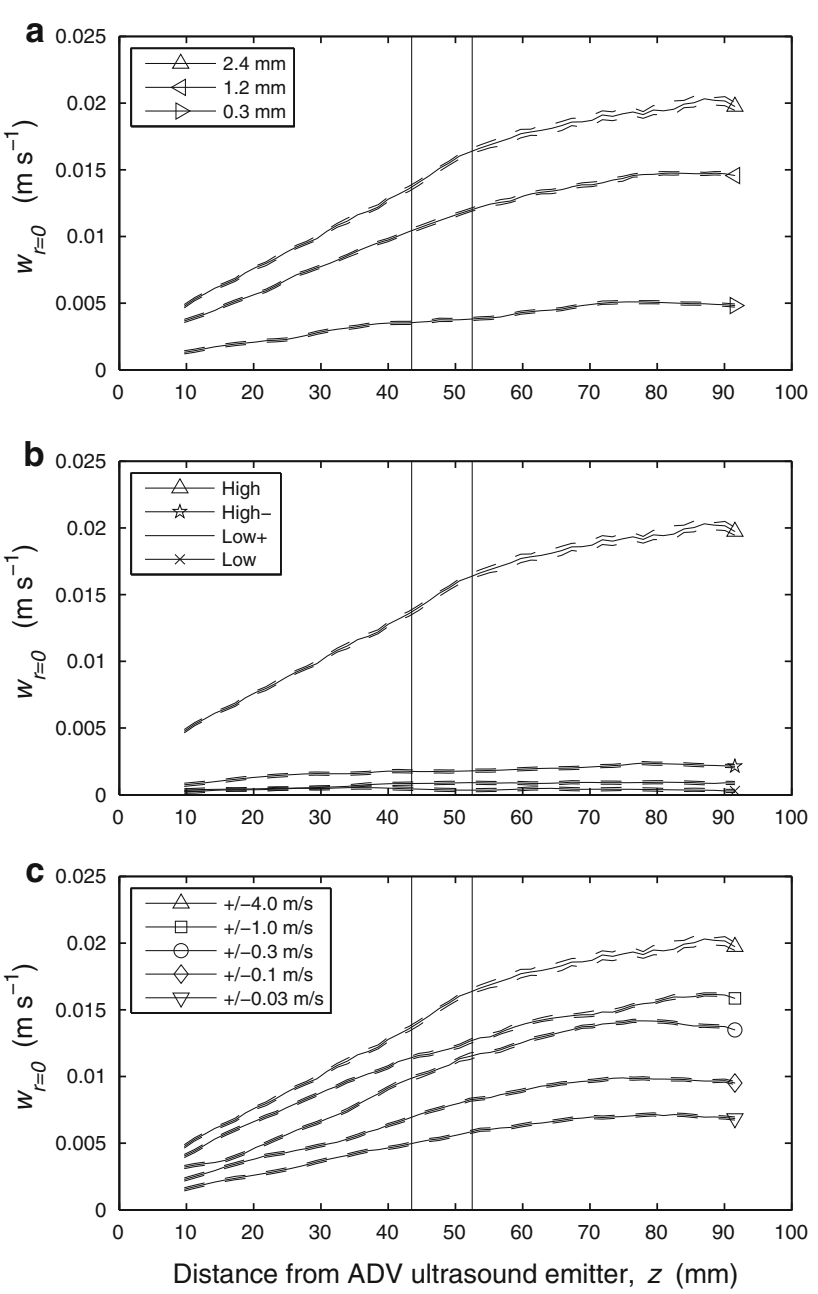

Fig. 3 Effect, as measured by PIV, of varying Vectrino transmit length, power, and nominal velocity range. Profiles of Vectrinoinduced acoustic streaming velocity along the transmit beam centerline, $w_{r=0}$, are shown for: a different transmit lengths with nominal velocity range and power level constant at $\pm 4.0 \mathrm{~ms}^{-1}$ and "high", respectively, $\mathbf{b}$ different power levels with nominal velocity range and transmit length constant at $\pm 4.0 \mathrm{~ms}^{-1}$ and $2.4 \mathrm{~mm}$, respectively, and c different nominal velocity ranges with transmit length and power level constant at $2.4 \mathrm{~mm}$ and "high", respectively, (Solid vertical lines show the approximate location of the sampling volume and dashed lines indicate uncertainty intervals)

space for each velocimeter, while the peak streaming location moves with configuration. The Vectrino's sample volume is centered $50 \mathrm{~mm}$ from its emit transducer while the ADVField's sample volume is centered $100 \mathrm{~mm}$ from its emit transducer. The Vectrino's peak streaming occurs beyond its sample volume (ranging from 60 to $90 \mathrm{~mm}$ from the emit transducer). The ADVField's peak velocity occurs between its sample volume and the ultrasound emitter (ranging from 70 to $90 \mathrm{~mm}$ from the emitter).

The differences between $w_{s}$ and $w_{p}$ are also related to the variation of the velocity in the direction perpendicular to the transmit beam, $w(r)$. At the center of the sampling

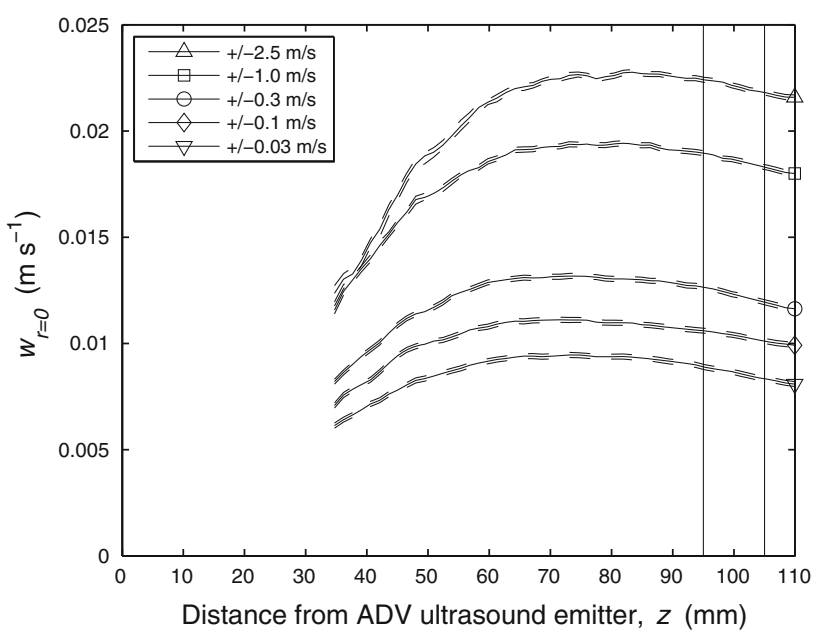

Fig. 4 Effect of varying nominal velocity range with the SonTek ADVField. The profile of acoustic streaming velocity along the transmit beam centerline, $w_{r=0}$, is shown for different nominal velocity ranges. (Solid vertical lines show the location of the sampling volume)

volume, radial profiles of axial velocity approximate Gaussian curves (Fig. 6), with widths $(\sigma)$ of 2.5 and $3.5 \mathrm{~mm}$ for the Vectrino and ADVField, respectively. As a result, the acoustic streaming velocity decreases significantly from the centerline to the edge of the sampling volume, thus $w_{s}<w_{p}$.

For all ADV configurations, radial velocities were generally an order of magnitude or more lower than vertical velocities and of the same order as background velocities. Image sets recorded with the ADV off were made just before most image sets of ADV-induced acoustic streaming (12 image sets in total, each with $11.5 \mathrm{~s}$ duration). From these images, we measured background vertical velocities as high as $0.4 \mathrm{~cm} \mathrm{~s}^{-1}$. Typically, background flow was much lower. The interquartile range of background vertical velocities, computed across the twelve image sets, averaged $0.012 \mathrm{~cm} \mathrm{~s}^{-1}$ over the field of view. The interquartile ranges for background horizontal velocities were of similar magnitude.

By comparing sample volume average vertical velocities, $w_{s}$, with velocity data collected by the Vectrino, we determine that for certain combinations of user-selectable settings, the Vectrino accurately measures the flow it induces. At high power, for nominal velocity ranges of $\pm 1.0 \mathrm{~m} \mathrm{~s}^{-1}$ or less, the Nortek Vectrino measured velocities that were statistically equivalent to PIV-based measurements $\left(w_{s}\right)$ or within $20 \%$ of the PIV-based measurements (Fig. 7). Selfmeasurement was not effective when the ADV was operated at lower power levels or at higher nominal velocity ranges. When operated in these configurations, the Vectrino reported median velocity measurements of approximately $0 \mathrm{~cm} \mathrm{~s}^{-1}$, with signal-to-noise ratios lower than $10 \mathrm{~dB}$ in all 
Table 2 Flows induced by two ADV models for different ADV configurations as observed via PIV

\begin{tabular}{|c|c|c|c|c|c|}
\hline $\begin{array}{l}\text { Nominal velocity } \\
\text { range }\left(\mathrm{ms}^{-1}\right)\end{array}$ & $\begin{array}{l}\text { Transmit } \\
\text { length (mm) }\end{array}$ & $\begin{array}{l}\text { Power } \\
\text { level }\end{array}$ & $\begin{array}{l}\text { Peak velocity } w_{p} \\
\left(\mathrm{~cm} \mathrm{~s}^{-1}\right)^{*}\end{array}$ & $\begin{array}{l}\text { Distance to peak } \\
\text { velocity } \\
\text { (mm below emitter) }\end{array}$ & $\begin{array}{l}\text { Average velocity } \\
\text { within ADV sampling } \\
\text { volume } w_{s}\left(\mathrm{~cm} \mathrm{~s}^{-1}\right)^{*}\end{array}$ \\
\hline \multicolumn{6}{|l|}{ Vectrino } \\
\hline 4.00 & 2.40 & High & 2.03 & 89 & 1.01 \\
\hline 1.00 & 2.40 & High & 1.62 & 90 & 0.88 \\
\hline 2.50 & 2.40 & High & 1.57 & 92 & 0.94 \\
\hline 4.00 & 1.20 & High & 1.47 & 90 & 0.81 \\
\hline 0.30 & 2.40 & High & 1.42 & 79 & 0.72 \\
\hline 0.30 & 1.80 & High & 1.17 & 87 & 0.63 \\
\hline 0.10 & 2.40 & High & 0.99 & 76 & 0.54 \\
\hline 0.03 & 2.40 & High & 0.76 & 84 & 0.44 \\
\hline 4.00 & 0.30 & High & 0.51 & 76 & 0.29 \\
\hline 0.03 & 1.20 & High & 0.42 & 70 & 0.26 \\
\hline 4.00 & 2.40 & High- & 0.24 & 84 & 0.14 \\
\hline 1.00 & 2.40 & High- & 0.17 & 67 & 0.13 \\
\hline 4.00 & 2.40 & Low+ & 0.09 & 82 & 0.07 \\
\hline 0.03 & 0.30 & High & 0.09 & 55 & 0.06 \\
\hline 0.03 & 2.40 & High- & 0.06 & 66 & 0.04 \\
\hline 4.00 & 2.40 & Low & 0.05 & 37 & 0.03 \\
\hline \multicolumn{6}{|l|}{ ADV field } \\
\hline 2.50 & NA & NA & 2.27 & 84 & 1.57 \\
\hline 1.00 & NA & NA & 1.94 & 83 & 1.29 \\
\hline 0.30 & NA & NA & 1.32 & 76 & 0.89 \\
\hline 0.10 & NA & NA & 1.11 & 73 & 0.71 \\
\hline 0.03 & NA & NA & 0.95 & 74 & 0.63 \\
\hline
\end{tabular}

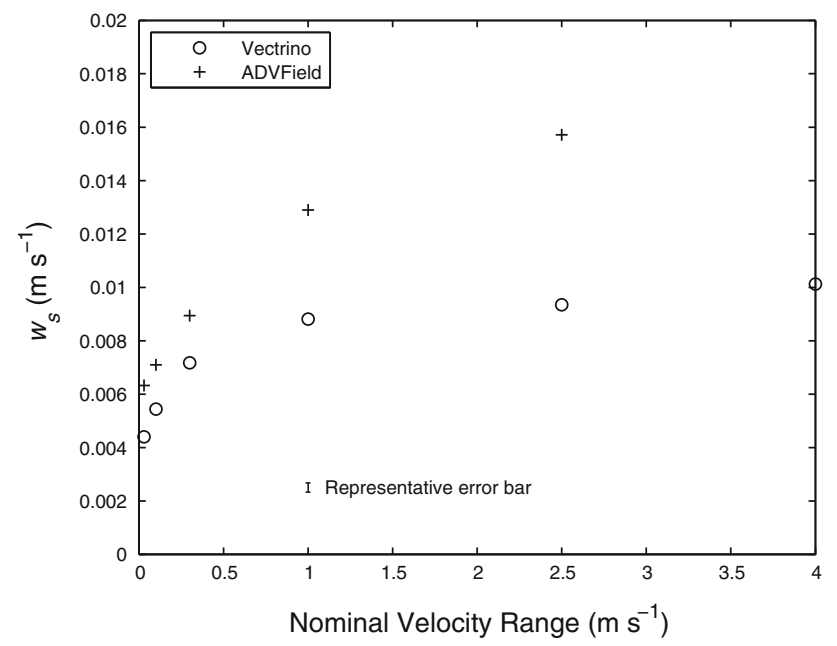

Fig. 5 Volume-averaged acoustic streaming velocity in the ADV sampling volumes, $w_{s}$, as measured by PIV

but one case. Self-measurement of the Vectrino-induced flow failed only when the SNR was substantially lower than $10 \mathrm{~dB}$ or the nominal velocity range setting was inappropriate (i.e. far greater than the range of observed velocities). The SonTek ADVField collected valid measurements of the flow it induced regardless of the nominal velocity range

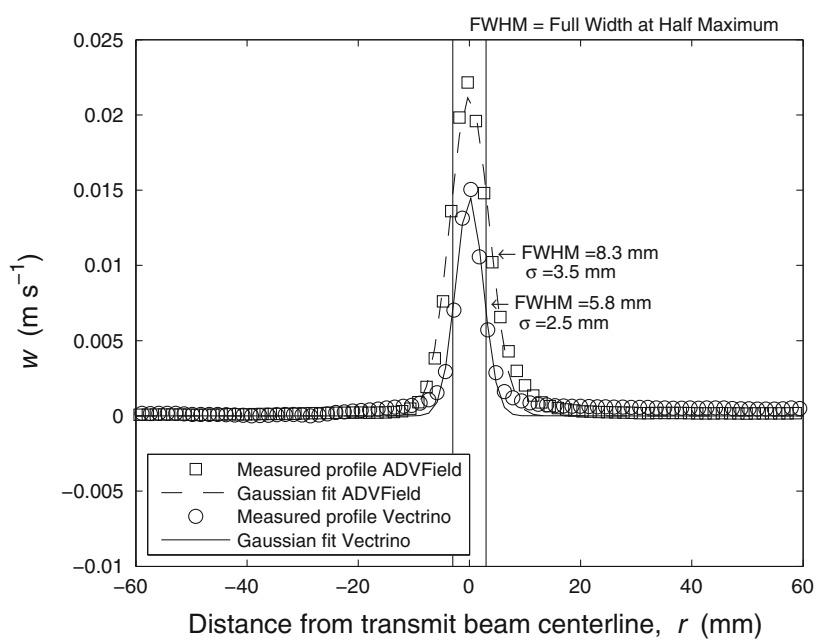

Fig. 6 Radial distribution of acoustic streaming velocities $w(r)$ for two ADV models at the vertical midpoint of their respective sampling volumes (radial extent of sample volumes shown as vertical lines). The settings generating maximum streaming velocities were selected for both velocimeters

setting. Self-measured flows were for all configurations within $50 \%$ of the PIV-based flow measurements. The discrepancies in the measurements were greatest for the two largest nominal velocity ranges (Fig. 8). Interestingly, the 


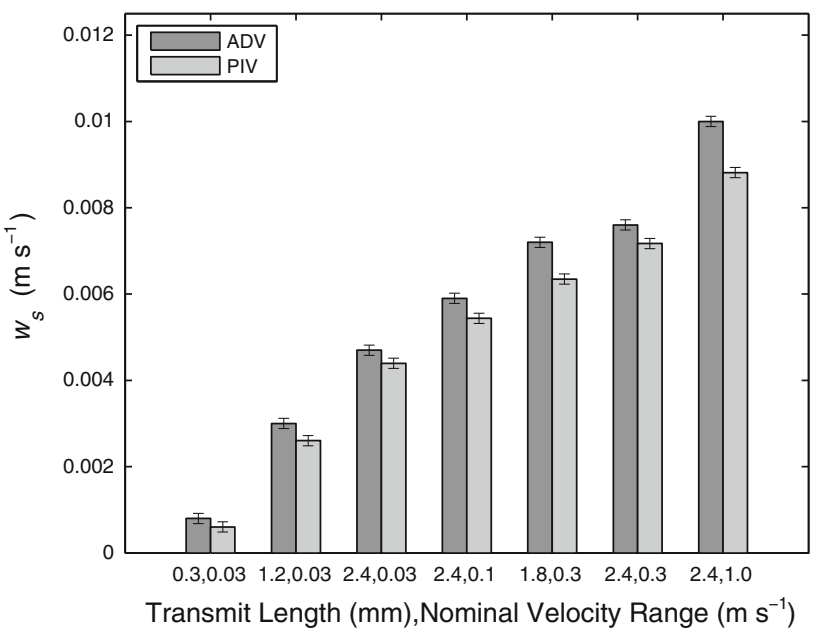

Fig. 7 Simultaneous ADV (Nortek Vectrino) and PIV measurements of Vectrino-induced acoustic streaming over its sampling volume, $w_{s}$, for different combinations of transmit length and nominal velocity range at high power

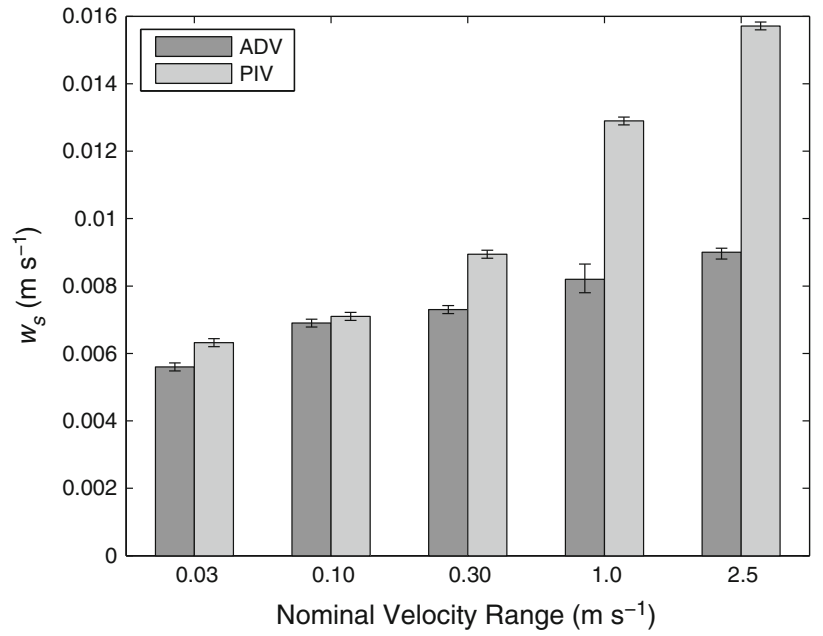

Fig. 8 Simultaneous ADV (SonTek ADVField) and PIV measurements of ADVField-induced acoustic streaming over the sampling volume, $w_{s}$, for different ADVField nominal velocity ranges

Nortek Vectrino self-measurements were consistently higher than PIV values, while SonTek ADVField selfmeasurements were consistently lower than PIV values. The ADVs' self-measurements could differ from the PIV values because our calculation of $w_{s}$ does not recreate the complex spatial averaging scheme used by the ADVs. When acoustic backscatter is of low strength, the Vectrino weights each localized velocity measurement (due to a single tracer particle) by the return signal strength (Atle Lohrmann, personal communication, 8/10/2010). When the backscattered signals are very strong, velocity data from all tracer particles in the sample volume are weighted equally. Thus, there is a continuum of spatial weighting functions that depend on particle type, particle loading, and ADV power level. Our
PIV-based estimates of sample volume velocity, $w_{s}$, use the simplest weighting scheme, i.e., a direct volume average corresponding to the case of large backscatter amplitude.

\section{Discussion}

Profiles of ADV-induced flow along the transmit beam axis $\left(w_{r=0}\right)$ (Figs. 3, 4) show the same features described in existing analytical models of acoustic streaming. These analytical solutions indicate acoustic streaming velocity increases with distance from the sound source ( $\mathrm{Wu}$ and $\mathrm{Du}$ 1993; Riley 2001). The observed flow increased with distance from the ADV emitter before peaking and beginning to decline at a distance between 30 and $90 \mathrm{~mm}$. Mitome et al. (1995) predicted such behavior, attributing it to momentum transport away from the ultrasound beam axis. The radial velocity distribution (Fig. 6) also agrees with the theoretical derivations of acoustic streaming velocity, which assume Gaussian profiles (Lighthill 1978).

Analytical solutions derived from Eqs. 1-3 predict that the magnitude of acoustic streaming varies either with transmitted sound amplitude, $a$, or with $a^{2}$ (Nyborg 1998; Mitome et al. 1995; Wu and Du 1993). Our data suggest a dependence on $a^{2}$. We found a linear relationship between $w_{p}$ and ADV time average power consumption for both the Vectrino and the ADVField (see Fig. 9). Power consumption data were collected with a wattmeter (Kill A Watt, P3 International) (Vectrino) or obtained from the operating manual (ADVField). For the Vectrino, time average power consumption, $P_{c}$, was measured while varying the userselectable power level at maximum transmit length and nominal velocity range. The ADVField's transmitted power is not directly user adjustable, and thus, the power usage

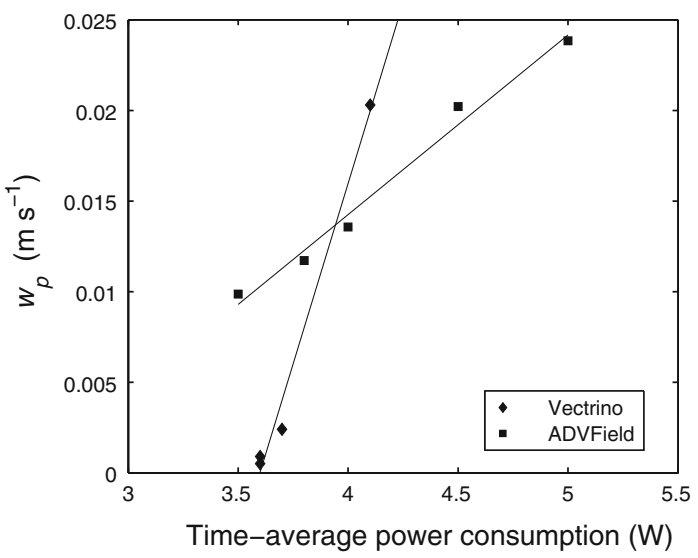

Fig. 9 Relationship between ADV time average power consumption and peak acoustic streaming velocity, $w_{\mathrm{p}}$. Vectrino time average power consumption was varied by adjusting the power level setting. ADVField time average power consumption was varied by adjusting the only setting typically adjusted: the nominal velocity range 
Table 3 The dependence of acoustic streaming velocity on sound amplitude and Vectrino power level

\begin{tabular}{llll}
\hline $\begin{array}{l}\text { Power } \\
\text { setting }\end{array}$ & $\begin{array}{l}\text { Sound } \\
\text { intensity } \\
\text { level }(\mathrm{dB})\end{array}$ & $\begin{array}{l}\text { Sound } \\
\text { amplitude, } \\
a(\mathrm{kPa})\end{array}$ & $\begin{array}{l}\text { Maximum Vectrino- } \\
\text { induced velocity } \\
w_{\mathrm{s}}(\mathrm{cm} / \mathrm{s})\end{array}$ \\
\hline High & 168 & 170 & $2.03 \pm 0.030$ \\
High- & 162 & 84 & $0.24 \pm 0.004$ \\
Low + & 156 & 42 & $0.09 \pm 0.002$ \\
Low & 150 & 21 & $0.05 \pm 0.001$ \\
\hline
\end{tabular}

data are less conclusive, as discussed further below. Additional support for the Vectrino's apparent linear relationship between transmitted sound power and acoustic streaming can be seen in the variation of transmitted sound intensity with power level setting (Table 3). When the transmit length and nominal velocity range settings are maximized, the highest Vectrino power level corresponds to time average sound intensity of approximately $168 \mathrm{~dB}$ (referenced to 1 microPascal at $1 \mathrm{~m}$ ) (Atle Lohrmann, personal communication, 7/14/2009). For continuous sound transmission from a 6-mm-diameter transducer through water, this intensity corresponds to a sound amplitude of $170 \mathrm{kPa}$. The difference between successively lower power levels is approximately $6 \mathrm{~dB}$ (Atle Lohrmann, personal communication, 7/14/2009), indicating that the minimum power level corresponds to a time average intensity of approximately $150 \mathrm{~dB}$ and an amplitude of $21 \mathrm{kPa}$ (for continuous sound). Sound amplitudes computed from these intensity values (assuming continuous sound and the reference pressure) show a clear quadratic relationship with $w_{p}$ $\left(R^{2}=0.99\right)$. These results also suggest that peak acoustic streaming velocity $w_{p}$ varies directly with sound power and hence sound amplitude squared $\left(a^{2}\right)$ not $a$. While the sound amplitudes calculated from time average intensity hold for continuous sound, the sound amplitudes for different power level settings would increase by the same factor for pulsed sound. Hence, the percent differences are correct even if the sound amplitudes are underestimated. When evaluating models with this data, it is important to consider that nonlinear sound propagation, which increases with amplitude, may obscure the relationship between amplitude and streaming velocity. Specifically, nonlinear ultrasound propagation, not accounted for in Eq. (2), transfers energy from the fundamental frequency to harmonics, which is more rapidly absorbed and thus magnifies acoustic streaming at higher power levels (Wu and Du 1993).

While the relationship between the ADVField's peak acoustic streaming velocity and its time average power consumption, $P_{c}$, seems to corroborate the dependence of $w_{p}$ on $a^{2}$ observed with the Vectrino, the ADVField power consumption is varied indirectly, via the nominal velocity range setting. This setting also changes the duty factor, in a way that is not publicly available. Thus, conclusions about the relationship between instantaneous transmitted power and acoustic streaming are not possible for the ADVField.

The effect of the duty factor on acoustic streaming in ADVs is of first-order importance, as evidenced by a comparison of additional Vectrino power consumption data, which prima facie seems to indicate no relationship between time average power consumption and acoustic streaming velocity. Minimizing the Vectrino nominal velocity range (and thus the duty factor) while maintaining the power at "high" reduces $P_{c}$ to $3.6 \mathrm{~W}$ and yields a $w_{p}$ of $0.76 \mathrm{~cm} \mathrm{~s}^{-1}$. When the Vectrino power level is set to "low+" and nominal velocity range is maximized, the time average power consumption is also $3.6 \mathrm{~W}$ yet $w_{p}$ drops to $0.09 \mathrm{~cm} \mathrm{~s}^{-1}$. This indicates the importance of other factors, namely duty factors and instantaneous transmitted power or sound amplitude. While these values are not publicly available, we infer their importance as follows.

For constant time average power, numerical models have shown that lower duty factors generate significantly higher acoustic streaming velocities (Wu and Du 1993). Smaller nominal velocity ranges, which correspond to lower pulse repetition frequencies and lower duty factors, in this investigation led to lower ADV-induced streaming. Shorter transmit lengths, which correspond to lower ratios of on to off time and hence lower duty factors, also led to lower acoustic streaming velocities when Vectrino power level remained constant. This behavior suggests that for the Vectrino, the instantaneous transmitted power and hence the sound amplitude remain constant regardless of pulse repetition frequency or transmit length. In other words, when pulse repetition frequency or transmit length is reduced, the time average sound power transmitted is also reduced. Indeed, power consumption measurements confirmed a drop in power consumption for lower nominal velocity ranges and transmit lengths. The same relationship between nominal velocity range and transmitted power holds true for ADVField, for which documentation explicitly states that lower duty factors use less time average power (SonTek/YSI 2001). Wu and Du's (1993) analytical solution implies that lower nominal velocity range settings generate faster acoustic streaming than would otherwise be predicted based on the time average power consumption. Instantaneous power and duty factor are thus the key variables that determine the magnitude of ADV-induced acoustic streaming, not time average power.

Observed ADV-induced streaming velocities fall within the range of those reported for medical ultrasound devices. A survey of diagnostic ultrasound equipment operating in distilled water reported maximum streaming velocities between 0.3 and $14 \mathrm{~cm} \mathrm{~s}^{-1}$ based on hot-film anemometry measurements (Starritt et al. 1989). A PIV-based study of 
ultrasonic lithotripters found axial streaming velocities up to $3 \mathrm{~cm} \mathrm{~s}^{-1}$ and radial velocities up to $1 \mathrm{~cm} \mathrm{~s}^{-1}$ (Choi et al. 2004). Another PIV study examined a $3.3-\mathrm{MHz}$ medical ultrasound device operating in continuous mode, observing maximum axial velocities of either 6 or $0.8 \mathrm{~cm} \mathrm{~s}^{-1}$ depending on the power setting selected (Cosgrove et al. 2001). Hartley (1995) tested a first generation ADV with adjustable sampling volume location and adjustable operating frequency in quiescent fluid (both water and blood) using the ADV itself. He reported sample volume average acoustic streaming velocities $\left(w_{s}\right)$ no greater than $1 \mathrm{~cm} \mathrm{~s}^{-1}$. The higher operating frequency (20 MHz) induced faster acoustic streaming than the lower operating frequency $(10 \mathrm{MHz})$. A nonlinear relationship between pulse repetition frequency and maximum streaming velocity was also observed.

When evaluating ADVs' self-measurement of acoustic streaming velocities (Figs. 7, 8), the disagreement between PIV and ADV data is particularly large at high nominal velocity settings. Use of higher nominal velocity ranges leads to higher ADV sampling error (McLelland and Nicholas 2000), and likely contributed to the greater disagreement at these ranges. Discrepancies between simultaneous ADV and PIV measurements may also be due the complex shape of the sampling volume, which was here approximated as a circular cylinder of diameter $6 \mathrm{~mm}$ for both ADVs.

The general conclusion of this work is that ADVinduced acoustic streaming can bias ADV measurements depending on the ADV configuration used. Our experiments in quiescent conditions do not, however, resolve the effect of ADV-induced flow on measurements in nonquiescent conditions. The nature of the ambient flow may influence the structure of the ADV acoustic stream and thus the way in which it affects measurements. We briefly consider two classes of ambient flow: unidirectional flow perpendicular to the ADV axis ("cross-flow") and homogeneous isotropic turbulence with no mean flow.

A cross-flow will advect the axial momentum away from the ADV emitter axis and sampling volume, potentially reducing the bias in ADV measurements. The measurements of Snyder and Castro (1999) showed ADVmeasured $w_{s}$ values were "substantially reduced" in the presence of a cross-flow of magnitude $0.9 \mathrm{~cm} \mathrm{~s}^{-1}$ when the ADV nominal velocity range was set to $3 \mathrm{~cm} \mathrm{~s}^{-1}$ (compared to a maximum $w_{s}$ of $0.7 \mathrm{~cm} \mathrm{~s}^{-1}$ in quiescent conditions). The streaming was reported to be absent at cross-flows of $2 \mathrm{~cm} \mathrm{~s}^{-1}$ or above. Counter to this line of reasoning, we note that successful ADV operation relies on the scattering of the emitted sound in the sampling volume. When emitted pulses continue to reach the sampling volume before being advected away (as required for accurate measurement), the spatial gradient created by sound absorption may continue to induce steady streaming. Consequently, we conjecture that cross-flow reduces but does not eliminate the ADV-induced acoustic streaming. To evaluate this, the effects of acoustic streaming would need to be separated from the effects of the ADV wake. The cross-flow velocity range between 0 and $2.0 \mathrm{~cm} \mathrm{~s}^{-1}$ remains to be studied and is important for cases such as wetlands and river backwaters.

To determine the effect of turbulence on acoustic streaming, we examined ADV and PIV data collected in an experiment that was performed to characterize a stirred turbulence tank (Variano and Cowen 2008). This apparatus had RMS turbulent velocities of approximately $4 \mathrm{~cm} \mathrm{~s}^{-1}$, with mean flows less than $0.5 \mathrm{~cm} \mathrm{~s}^{-1}$. The data on mean velocities show strong evidence that acoustic streaming exists and influences the ADV measurements despite the turbulent shearing motions (Fig. 10). The statistically stationary flow was measured with a Nortek Vectrino ADV and then by PIV (with the ADV probe removed). The ADV probe was mounted in two orientations: first with the ultrasound emitter axis oriented toward the negative $z$-direction (tank coordinates) and then with emitter axis oriented toward the positive $x$-direction. Comparing the three measurements suggests that ADV induces a velocity of roughly $0.2 \mathrm{~cm} \mathrm{~s}^{-1}$ in the direction parallel to the emitter axis. That is, PIV and ADV measurements agree in any direction other than the ADV's axial direction and the ADV velocities shift when the orientation is shifted. The turbulence is nearly isotropic and the mean flows small; thus, we expect that the effect is not due to altered ADV wakes. While noise levels in ADV-measured axial

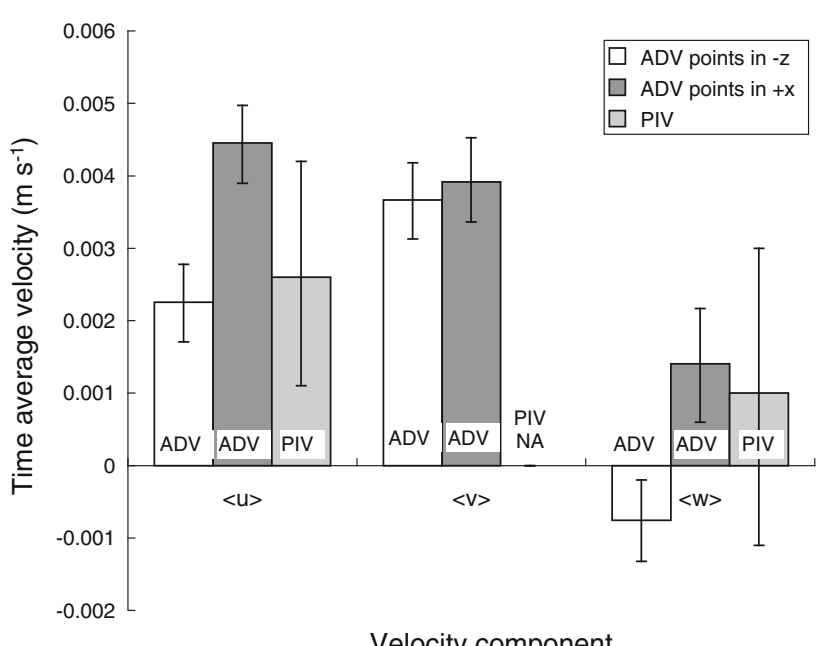

Fig. 10 Time-averaged mean velocities in a turbulent stirred tank as measured by PIV and an ADV oriented in two different directions. Discrepancies between measurements suggest that acoustic streaming persists and biases ADV measurements despite turbulent flow and cross-flow 
velocities differ from noise levels in the radial velocities due to probe geometry, this phenomenon does not affect mean flow measurements (Voulgaris and Trowbridge 1998). The apparent effect of acoustic streaming on the sample volume average flow velocity is roughly $25 \%$ of that which we would expect in a quiescent flow, where a Vectrino with the same settings (Nominal velocity range $= \pm 1.00 \mathrm{~m} \mathrm{~s}^{-1}$, Transmit length $=2.4 \mathrm{~mm}$, Power Level $=$ High) would produce a $w_{s}$ of approximately $0.8 \mathrm{~cm} \mathrm{~s}^{-1}$ (see Fig. 5). This evident persistence of acoustic streaming despite strong turbulent shear lends some support to our hypothesis that acoustic streaming will persist in a cross-flow. In this respect, it is also of interest to note that there is indeed a cross-flow (albeit a weak one) in this data.

The extent to which ADV acoustic streaming affects ADV data quality depends on a combination of four factors: (1) the internal (non-adjustable) ADV specifications, (2) user-selected configuration, (3) ambient conditions, and (4) the velocity statistics of interest. The internal ADV specifications that influence how acoustic streaming impacts measurement are the ultrasound frequency and the location of the sampling volume. Devices using higher frequency ultrasound such as the SonTek 16-MHz ADVOcean are expected to generate higher streaming velocities. The user-adjustable range of time average transmitted power levels and duty factors will determine the extent and pattern of streaming, though the user's range of choices are typically restricted by other demands of the measurement. That is, the user's selection of a nominal velocity range is typically governed by the expected flow conditions, while selection of the power level and transmit length is governed by particle density in the flow. Ambient conditions affecting streaming velocity include cross-flow velocity and turbulence, although the interaction between these and acoustic streaming is not yet well characterized. Temperature and particle density will likely affect the streaming velocities because they influence ultrasound absorption, the primary driving force behind acoustic streaming. In pure water, sound attenuation decreases with increasing temperature (up to $74^{\circ} \mathrm{C}$ ) (Kuttruff 1991), and thus we expect larger acoustic streaming at colder temperatures. The shape of suspended solids (Hay 1991) and the concentration and composition of dissolved substances also affect ultrasound absorption (Kuttruff 1991); the effect of these factors on acoustic streaming may be of great importance to studies that use ADVs to measure sediment fluxes.

While the user typically has few options for changing the above three factors, they may have control over the orientation of the velocimeter probe. By carefully choosing the ADV orientation with respect to the velocity statistics of interest, one can reduce the significance of acoustic streaming for flow measurements. The very low radial velocities measured here and in most observational studies of ultrasound-induced streaming suggest that measurement of velocity components perpendicular to the ADV emitter axis is negligibly impacted (higher order statistics such as Reynolds' stresses may be affected). Thus, to minimize bias from acoustic streaming, the ADV's emitter axis should be aligned perpendicular to the flow direction of greatest interest. Unfortunately, due to the geometry of the probe, the component of velocity parallel to the emitter axis is the one most precisely measured by the ADV (SonTek 1997). Measurements of velocities parallel to the emitter axis also exhibit many times less noise than measurements of velocities perpendicular to the emitter axis (Lohrmann et al. 1994; Nikora and Goring 1998; Cea et al. 2007). Hence, as far as ADV probe orientation, there is a trade-off between precision and accuracy in cases where acoustic streaming is non-negligible.

If the experimental details cannot be altered to eliminate the bias due to acoustic streaming, the magnitude of the bias should be quantified as best as possible. The simplest way to quantify acoustic streaming bias is to use values reported here for $w_{s}$, with the important caveat that different water quality parameters, flows, and ADV specifications can cause significant differences in acoustic streaming velocities. Vectrino measurements were collected at 24 degrees $C$ and ADVField measurements at $21^{\circ} \mathrm{C}$. The loading of the 11-micron glass spheres used as PIV tracer particles (estimated to be $1 \mathrm{mg} \mathrm{l}^{-1}$ ) was optimized for PIV. Alternative options for estimating the induced flow include (in approximate order of increasing cost and difficulty) measuring streaming in a quiescent water sample with the ADV itself, comparing velocity statistics for different ADV orientations, predicting streaming velocity via analytical or numerical models (e.g. Wu and Du 1993; Kamakura et al. 1996), or measuring streaming directly with an alternate flow measurement technology.

The flow chart in Fig. 11 organizes the results of our analysis to help ADV users identify whether acoustic streaming bias is significant for a particular application and, if so, choose an option for characterizing the bias. Common environmental flows in which acoustic streaming may represent a significant source of bias include wetlands, river backwaters, sedimentation tanks, and tidal flows near slack tide. In low-flow situations such as these, acoustic streaming bias is likely outside manufacturer-specified error bounds of $0.5 \%$ of the measured value or $\pm 1 \mathrm{~mm} \mathrm{~s}^{-1}$ for the Vectrino (Nortek 2009) and 1\% of the measured velocity or $\pm 0.25 \mathrm{~cm} \mathrm{~s}^{-1}$ for the ADVField (SonTek 1997) (Table 4). In fast boundary layer flows, the wake generated by some ADV probes can cause vertical velocities of approximately $2 \%$ of the horizontal flow velocity (Snyder and Castro 1999), potentially overshadowing acoustic streaming-related error. 
Fig. 11 A flow chart for identifying when acoustic streaming may bias ADV measurements and selecting an option for characterizing acoustic streaming velocity in these situations

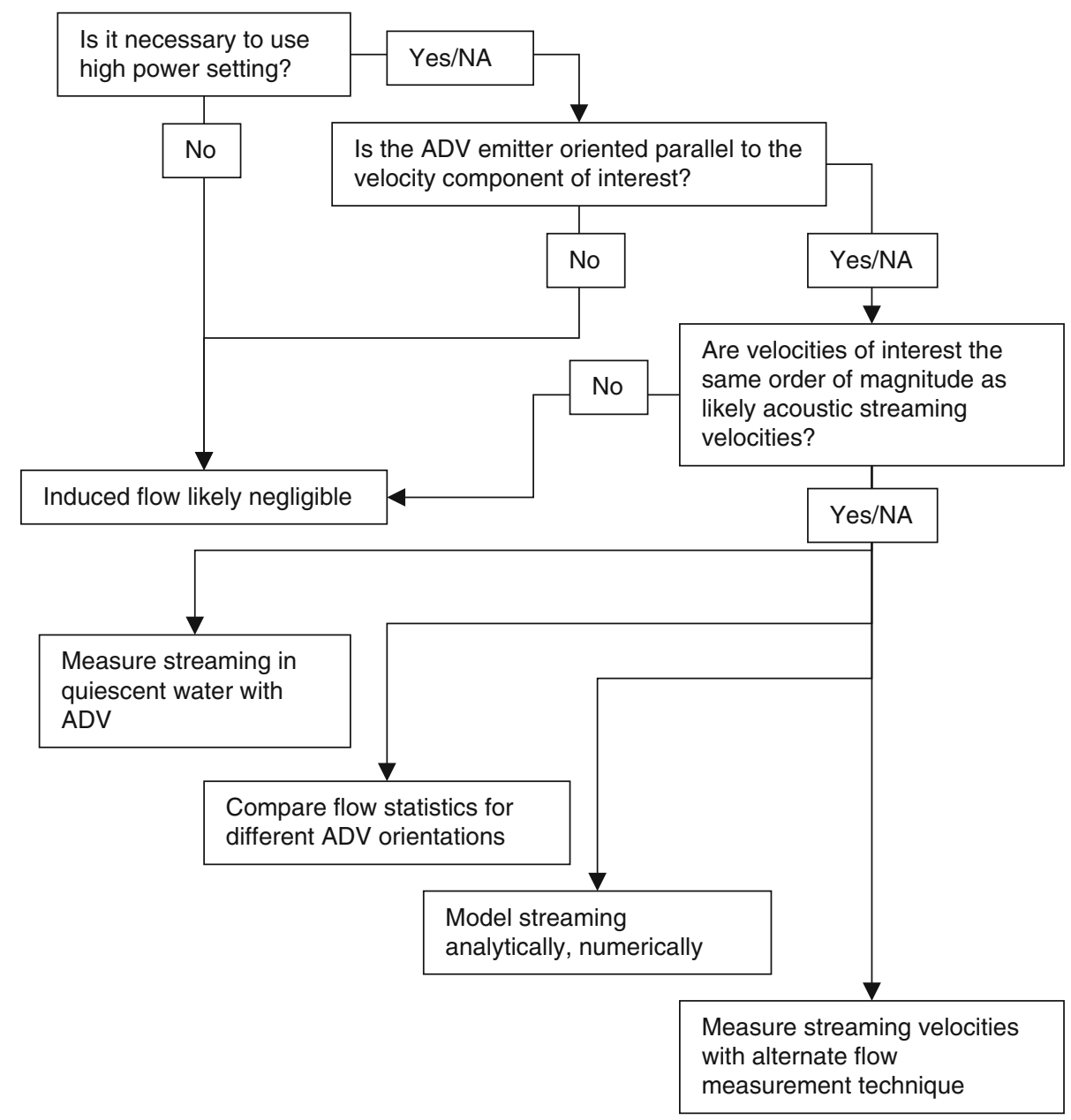

Table 4 The error due to acoustic streaming as a percentage of measured velocity for different nominal velocity ranges

\begin{tabular}{|c|c|c|c|c|c|}
\hline $\begin{array}{l}\text { Nominal velocity } \\
\text { range } \\
\left(\mathrm{ms}^{-1}\right)\end{array}$ & $\begin{array}{l}\text { Vertical velocity range (the maximum } \\
\text { vertical velocity optimally measured } \\
\text { in this nominal. velocity range) } \\
\left(\mathrm{ms}^{-1}\right)\end{array}$ & $\begin{array}{l}\text { Minimum vertical velocity } \\
\text { optimally measured at } \\
\text { this nominal velocity range } \\
\left(\mathrm{ms}^{-1}\right)\end{array}$ & $\begin{array}{l}\text { Sample volume } \\
\text { average acoustic } \\
\text { streaming velocity, } w_{s} \\
\left(\mathrm{~ms}^{-1}\right)\end{array}$ & $\begin{array}{l}\text { Minimum } \\
\text { acoustic } \\
\text { streaming } \\
\text { error }(\%)\end{array}$ & $\begin{array}{l}\text { Maximum } \\
\text { acoustic } \\
\text { streaming } \\
\text { error }(\%)\end{array}$ \\
\hline \multicolumn{6}{|l|}{ Vectrino } \\
\hline 0.03 & 0.08 & NA & 0.0044 & 5.5 & $>5.5$ \\
\hline 0.10 & 0.13 & 0.08 & 0.0054 & 4.2 & 6.8 \\
\hline 0.3 & 0.27 & 0.13 & 0.0072 & 2.7 & 5.5 \\
\hline 1.0 & 0.54 & 0.27 & 0.0088 & 1.6 & 3.3 \\
\hline 2.5 & 0.94 & 0.54 & 0.0094 & 0.99 & 1.7 \\
\hline 4.0 & 1.5 & 0.94 & 0.010 & 0.68 & 1.1 \\
\hline \multicolumn{6}{|l|}{ ADV field } \\
\hline 0.03 & 0.08 & NA & 0.0063 & 7.9 & $>7.9$ \\
\hline 0.10 & 0.15 & 0.08 & 0.0071 & 4.7 & 8.9 \\
\hline 0.30 & 0.3 & 0.15 & 0.0089 & 3.0 & 5.9 \\
\hline 1.0 & 0.75 & 0.3 & 0.013 & 1.7 & 4.3 \\
\hline 2.5 & 0.9 & 0.75 & 0.016 & 1.7 & 2.1 \\
\hline
\end{tabular}

Percent error values assume: (a) quiescent flow acoustic streaming magnitudes and (b) a nominal velocity range chosen for optimal measurement of vertical velocity 


\section{Conclusions}

In quiescent water, the SonTek 10-MHz ADVField and the Nortek Vectrino were observed to induce acoustic streaming along the transmit beam, thereby altering the velocity within the sampling volume. The flow is primarily in the axial direction and increases with distance from the ultrasound emitter before reaching a peak as high as $2 \mathrm{~cm} \mathrm{~s}^{-1}$ at a distance of $6-9 \mathrm{~cm}$ from the emitter. Analytical models for acoustic streaming from the literature and a comparison between the two ADVs suggest streaming velocities are proportional to the peak instantaneous transmitted ultrasound power and the duty factor. Peak instantaneous transmitted power varies with the useradjustable power level setting (Vectrino only), and duty factor depends on both the transmit length and nominal velocity range settings (both ADVs).

If ambient flow velocities are of the order of potential acoustic streaming velocities, then acoustic streaming may represent a significant source of bias. Measurements most likely biased by acoustic streaming are those of velocity components aligned with the ADV emitter axis in low flows. The effect is present, though of lesser magnitude, in turbulent flows and cross-flows.

A range of options exist for accounting for streaming when it is unavoidable, including measurement of streaming magnitudes with the ADV itself and analytical or numerical modeling. We evaluate the quality with which the ADVs measure the acoustic streaming they induce, finding ADV measurements agree to within $20 \%$ when the velocity settings are appropriate for the streaming magnitude in question. Analytic models for acoustic streaming describe the general form of the ADVs' induced flow well, although special consideration is necessary due to the pulsed nature of ADVs' ultrasound transmission. The measurements presented here are used to guide the application and refinement of the existing models to more accurately describe ADVs.

Acknowledgments We would like to thank the University of California, Berkeley for financial support, Andreas Brand and Audric Collignon for laboratory and intellectual contributions, and Atle Lohrmann for indispensable clarifications of ADV principles of operation. We also thank three anonymous reviewers for thoughtful comments on the manuscript.

Open Access This article is distributed under the terms of the Creative Commons Attribution Noncommercial License which permits any noncommercial use, distribution, and reproduction in any medium, provided the original author(s) and source are credited.

\section{References}

Cea L, Puertas J, Pena L (2007) Velocity measurements on highly turbulent free surface flow using ADV. Exp Fluids 42:333-348
Choi MJ, Doh DH, Cho CH, Kang KS, Paeng DG et al (2004) Visualization of acoustic streaming produced by lithotripsy field using a PIV method. J Phys Conf Ser 1:217-223

Cosgrove JA, Buick JM, Pye SD, Greated CA (2001) PIV applied to Eckart streaming produced by a medical ultrasound transducer. Ultrasonics 39:461-464

Elgar S, Raubenheimer B, Guza RT (2005) Quality control of acoustic Doppler velocimeter data in the surfzone. Meas Sci Technol 16:1889-1893

Faraday M (1831) Acoustic streaming. Philos Trans R Soc Lond 121:299-340

Hartley CJ (1995) Doppler measurement of acoustic streaming. In: Proceedings of the 1995 IEEE Ultrasonics Symposium, IEEE Publishing, pp 1537-1540

Hay AE (1991) Sound scattering from a particle-laden, turbulent jet. J Acoust Soc Am 90:2055-2074

Kamakura T, Sudo T, Matsuda K, Kumamoto Y (1996) Time evolution of acoustic streaming from a planar ultrasound source. J Acoust Soc Am 100:132-138

Kaye GWC, Laby TH (1986) Tables of physical and chemical constants. Longman, New York

Kumar A, Gogate PR, Pandit AB (2007) Mapping of acoustic streaming in sonochemical reactors. Ind Eng Chem Res $46: 4368-4373$

Kuttruff H (1991) Ultrasonics: fundamentals and applications. Elsevier Applied Science, New York

Lhermitte R, Serafin R (1984) Pulse-to-pulse coherent Doppler sonar signal processing techniques. J Atmos Ocean Technol 1:293-308

Lighthill J (1978) Acoustic streaming. J Sound Vib 61:391-418

Lohrmann A, Cabrera R, Kraus NC (1994) Acoustic-Doppler velocimeter (ADV) for laboratory use. In: Fundamentals and advancements in hydraulic measurements and experimentation proceedings. ASCE publications, NY, pp 351-365

Makarov S, Semenova N, Smirnov V (1989) Acoustic streaming model for an intense sound beam in free space. Fluid Dyn 24:823-826

McLelland SJ, Nicholas AP (2000) A new method for evaluating errors in high-frequency ADV measurements. Hydrol Process 14:351-366

Mitome H, Kozuka T, Tuziuti T (1995) Effects of nonlinearity in development of acoustic streaming. Jpn J Appl Phys 34:2584-2589

Nepf HM (1999) Drag, turbulence, and diffusion in flow through emergent vegetation. Water Resour Res 35:479-489

Nikora VI, Goring DG (1998) ADV measurements of turbulence: can we improve their interpretation? J Hydraul Eng 124(6): 630-634

Nortek AS (2009) Vectrino velocimeter manual. Nortek AS, Norway

Nyborg WL (1998) Acoustic streaming. In: Hamilton MF, Blackstock DT (eds) Nonlinear Acoustics. Academic Press, Chestnut Hill

Riley N (1997) Acoustic streaming. In: Crocker MJ (ed) Encyclopedia of Acoustics. Wiley, New York

Riley N (2001) Steady streaming. Annu Rev Fluid Mech 33:43-65

Snyder WH, Castro IP (1999) Acoustic Doppler velocimeter evaluation in stratified towing tank. J Hydraul Eng 125:595-603

SonTek (1997) Acoustic Doppler Velocimeter (ADV) principles of operation. SonTek, San Diego

SonTek/YSI (2001) ADVField operation manual. SonTek/YSI, San Diego

Starritt H, Duck F, Humphrey V (1989) An experimental investigation of streaming in pulsed diagnostic ultrasound beams. Ultrasound Med Biol 15:363-373

Variano EA, Cowen EA (2008) A random-jet-stirred turbulence tank. J Fluid Mech 604:1-32 
Voulgaris G, Trowbridge JH (1998) Evaluation of the acoustic Doppler velocimeter (ADV) for turbulence measurements. J Atmospheric Ocean Technol 15:272-289
Wu J, Du G (1993) Acoustic streaming generated by a focused Gaussian beam and finite amplitude tonebursts. Ultrasound Med Biol 19:167-176 